\title{
Dramapädagogische Elemente im Leseförderprojekt „Mehrsprachiges Lesetheater" (MELT)
}

\author{
Angelika Ilg/Sabine Kutzelmann/Ute Massler/Klaus \\ Peter/Kerstin Theinert
}

\begin{abstract}
Zusammenfassung
Individuelle Mehrsprachigkeit und ungenügende Lesekompetenzen sind gesellschaftlich wie auch bildungspolitisch relevante Themen in den vier Ländern (Deutschland, Österreich, Schweiz und Luxemburg) des EUProjektes MELT (2014-2017), in dessen Zentrum die Entwicklung des didaktisch-methodischen Designs „Mehrsprachiges Lesetheater“ steht (siehe http://melt-multilingual-readers-theatre.eu). Das im Projekt zu entwickelnde Design wird nach dem Design-Based Research-Ansatz (DBR) erarbeitet (vgl. Van den Akker et al. 2006).

Lesetheater gehört zu den Lautleseverfahren, die im Bereich der Leseflüssigkeit signifikante Verbesserungen hinsichtlich der korrekten Worterkennung, der Lesegeschwindigkeit und der Prosodie erzielen sowie einen hohen motivationalen Effekt haben (vgl. Young \& Rasinski 2009: 10). Konkret werden im Projekt literarische Texte in mehrsprachige dialogische Lesetheaterstücke umgewandelt, je nach landesspezifischem Praxiskontext in einer anderen Sprachenkombination aus Schul-/Fremd- und Migrationssprachen. Diese bilden die Grundlage für das Training des gestaltenden Vorlesens in kooperierenden Schülerarbeitsgruppen wie auch für die im Klassenverband abschließende Aufführung von Lesetheatern. In der Trainingsphase üben die SchülerInnen ihre einzelnen Leserollen, indem sie diese wiederholt laut oder halblaut gestaltend lesen. Gerade in dieser Probenphase kommen auch theater- und dramapädagogische Elemente zum Einsatz, wenn sich SchülerInnen beispielsweise mit der Gefühls- und Gedankenwelt der von ihnen dargestellten Figur auseinandersetzen oder sich mithilfe von dramapädagogischen Übungen den Inhalt der zu lesenden Szene erarbeiten. Der Beitrag widmet sich deshalb unter anderem der Frage, wie und unter welchen Bedingungen dramapädagogische Elemente effektiv in ein Unterrichtsdesign für mehrsprachiges Lesetheater integriert werden können.
\end{abstract}




\section{Einleitung}

Der vorliegende Beitrag beschäftigt sich mit mehrsprachigem Lesetheater einer neuen und innovativen Form einer mehrsprachigen inszenierten Lernumgebung, die darauf abhebt, die Leseflüssigkeit und Lesemotivation von SchülerInnen in den Schulstufen 5 bis 8 in der Schul- und Fremdsprache zu fördern sowie - aufgrund der sprachenübergreifenden Gestaltung - auch die Mehrsprachigkeitskompetenz der Lernenden zu erhöhen. Konkret wird in diesem Beitrag der Frage nachgegangen, welche Funktionen dramapädagogischen Elementen in diesem mehrsprachigen didaktisch-methodischen Design zukommen und inwiefern sie dazu beitragen können, die mit dem Unterrichtsdesign verfolgten Ziele zu erreichen.

Mit dem Begriff Dramapädagogik soll hier auf den englischen Begriff Drama in education (Schewe 1993: 13) Bezug genommen werden, der „die Nutzung dramatischer bzw. theatralischer Mittel für pädagogische Zwecke“ (Kessler 2008: 37) bezeichnet. Mithilfe dramatischer Übungen und Techniken sollen „fiktive dramatische Situationen geschaffen werden, die es ermöglichen, unterschiedlichste Aspekte der menschlichen Erfahrung zu erkunden und zu durchleuchten“ (vgl. ebd.). Spätestens seit den 1990er Jahren hat die Dramapädagogik im kommunikativ orientierten Sprachunterricht ihren festen Platz - dramapädagogische Konzepte in der Fremdsprachendidaktik gelten als eine „Weiterführung der kommunikativen Didaktik“ (Schewe 2011: 22). Die Dramapädagogik sieht ihr Potenzial vor allem darin, „das Fremdsprachenlernen erfahrungsbezogen und lernerorientiert zu gestalten" (Küppers et al. 2011: 8). Bei der Arbeit mit dramapädagogischen Elementen im Fremdsprachenunterricht sollen zudem die Vorstellungskraft und die Kreativität gefördert sowie das Reflexionsvermögen geschult werden (vgl. Bonnet \& Küppers 2011). Darüber hinaus sind das soziale Lernen, die Entwicklung von Kooperations-, Recherche- und Problemlösefähigkeiten und die Verbesserung der kommunikativen Kompetenz von Bedeutung. Diese Komponenten können SchülerInnen zu kritischen, selbstständigen Denkern erziehen. Vorrangiges Ziel ist die Persönlichkeitsentwicklung (vgl. ebd.). Ergänzend dazu listen Maley \& Duff (1978/2005: 1) weitere Begründungen für den Einsatz von drama techniques in language learning auf:

It integrates verbal and nonverbal aspects of communication, thus bringing together both mind and body, and restoring the balance between physical and intellectual aspects of learning. It fosters self-awareness (and awareness of others), self-esteem and confidence; and through this, motivation is developed.

Da MELT zur Förderung der Leseflüssigkeit und Lesemotivation ähnliche Ziele verfolgt, lassen sich dramapädagogische Methoden gut in das Unterrichtsdesign einbinden. Vor diesem Hintergrund werden in Abschnitt 2 zunächst die Zielsetzungen des Unterrichtsdesigns sowie der Forschungsansatz, an dem sich die Entwicklungsarbeit für das Design orientiert, skizziert. In Abschnitt 3 
wird sodann anhand konkreter Beispiele der Entwicklungsprozess des Designs illustriert, während in Abschnitt 4 die Funktion dramapädagogischer Elemente im Design erörtert wird, bevor der Beitrag mit einem Fazit und einem Ausblick auf die Weiterentwicklung des Designs in Abschnitt 5 schließt.

\section{Das Unterrichtsdesign MELT - „Mehrsprachiges Lesetheater"}

Das Unterrichtsdesign MELT wird im Rahmen des Erasmus+-Projektes „Mehrsprachiges Lesetheater zur Förderung von Lesemotivation und Leseflüssigkeit (2014-2017)“entwickelt. Am Projekt sind die drei Pädagogischen Hochschulen St. Gallen, Vorarlberg und Weingarten, das SCRIPT Inno des luxemburgischen Bildungsministeriums sowie je eine Kooperationsklasse in den Partnerländern Deutschland, Luxemburg, Österreich und der Schweiz (insgesamt ca. 80 SchülerInnen) beteiligt.

Das im Projekt entwickelte didaktisch-methodische Design reagiert auf drei Herausforderungen im Bereich der Schul- und Fremdsprachendidaktik:

- Die Ergebnisse der großen Leseleistungsstudien der letzten Dekade haben aufgezeigt, dass es insbesondere in der Sekundarstufe I in den MELT-Projektländern Defizite in der Lesekompetenz in Schul- und Fremdsprache gibt - dies gilt insbesondere für Lernende mit schwachem sozioökonomischem Hintergrund (vgl. OECD 2014: 187-217, OECD 2011: 44-46).

- In den Curricula der Partnerländer wird seit mehreren Jahren anstelle eines isolierten Sprachenlernens die stärkere Vernetzung von Schul- und Fremdsprache gefordert (vgl. u. a. Europarat 2001; Herdina/Jessner 2002; Allgäuer-Hackl/Jessner 2013). Es gibt bisher jedoch nur wenige konkrete Vorschläge dafür, wie sprachübergreifender Unterricht konkret umgesetzt werden kann.

- Die Förderung der individuellen Mehrsprachigkeit ist ein erklärtes Ziel der EU-Bildungspolitik: Mindestens 75 \% der SchülerInnen der Sekundarstufe I sollen mindestens zwei Fremdsprachen lernen (vgl. European Commission 2012: 33 und 35).

Aus diesen drei bildungspolitischen Herausforderungen, die miteinander verzahnt sind, ergeben sich für die Bildungswissenschaften und Fachdidaktiken eine Reihe von Fragen, auf die es keine einfachen oder vorgefertigten Antworten gibt. Um ein Unterrichtsdesign zu entwickeln, das versucht, in angemessener Weise auf die oben genannten Herausforderungen zu reagieren, orientiert sich die Forschungs- und Entwicklungsarbeit im Projekt MELT an den Grundsätzen von Design-Based Research, dessen Stärke in der engen Zusammenarbeit von Forschenden und PraktikerInnen liegt. 


\subsection{Forschungsansatz: Design-Based Research}

Unter Educational Design Research oder Design-Based Research (DBR) versteht man

... the systematic study of analyzing, designing and evaluating educational interventions in order to solve complex educational problems for which no ready-made solutions are available and to gain insight in key design principles (Nieveen 2010: 89).

Design-Based Research zielt immer darauf ab, Forschung und Entwicklung miteinander zu verbinden: Einerseits werden in DBR-Entwicklungsprozessen Lösungen (sog. Unterrichtsdesigns) für komplexe unterrichtsbezogene Probleme entwickelt; andererseits soll im Zuge des Entwicklungsverfahrens das theoretische Wissen über die mit dem Design zusammenhängenden Lehr- und Lernprozesse sowie über das Design-Entwicklungsverfahren selbst erweitert werden (vgl. Van den Akker 2006: 4; Plomp 2010: 12). Van den Akker et al. (2006: 5) nennen fünf Merkmale von Design-Based Research, die im Projekt MELT umgesetzt werden:

- Interventionsorientierung: Das methodisch-didaktische Design reagiert auf eine aktuelle Frage, die sich aus der Schulrealität und -praxis ergibt.

- Zyklische Vorgehensweise: Der Entwicklungsprozess durchläuft mehrere Phasen der Erprobung, Evaluation und fortlaufenden Überarbeitung.

- Prozessorientierung: Im Fokus des Projekts stehen u. a. auch die durch das Design initiierten Lehr- und Lernprozesse.

- Praxisorientierung: Ziel ist es, ein Unterrichtsdesign zu entwickeln, das in der Praxis angenommen und eingesetzt wird.

- Theorieorientierung: Die Entwicklung des Designs nimmt ihren Ausgangspunkt in der Theorie; der Entwicklungsprozess selbst führt wiederum zu theoretischen Erkenntnissen im Bereich der Lehr- und Lernforschung.

Diesen Grundsätzen entsprechend wird das Unterrichtsdesign in der Entwicklungsphase an den einzelnen Kooperationsschulen jeweils in einer Klasse in insgesamt sechs Erprobungszyklen erprobt. Die Erprobungen finden jeweils im Klassenverband im Regelunterricht statt. Die Erfahrungen, die hierbei gemacht werden, und die Rückmeldungen der Kooperationslehrpersonen bilden nach jedem Erprobungszyklus die Grundlage für die gemeinsame Überarbeitung und Optimierung des Designs. Im Zuge dieses MELT-Entwicklungsprozesses soll die Frage beantwortet werden, wie ein Unterrichtsdesign zu mehrsprachigem Lesetheater gestaltet werden sollte, das die Leseflüssigkeit und Lesemotivation fördert und zugleich von Lehrpersonen und SchülerInnen in der Praxis gut angenommen wird. 


\section{2 „Mehrsprachiges Lesetheater" und dessen Förderdimensionen}

Lesetheater ist eine dramatische Verfahrensweise, bei der - wie der Name vermuten lässt - das szenische Lesen von Texten im Vordergrund steht. Es geht also nicht darum, einen Text frei wiederzugeben oder zu inszenieren, sondern diesen möglichst flüssig gestaltend zu lesen. Das Lesetraining findet weniger während der Aufführung, sondern vielmehr im Zuge der Vorbereitung auf die Aufführung des Lesetheaters statt. In dieser Phase werden die einzelnen Leserollen von den SchülerInnen wiederholt laut oder halblaut gestaltend gelesen. Gerade von diesem mehrfach wiederholten lauten, prosodischen Lesen erhofft sich die Lesedidaktik Effekte in Hinblick auf die Leseflüssigkeit der SchülerInnen. Lesetheater gelten deshalb in der Lesedidaktik als eine Spielart von Lautleseverfahren, die in der Unterrichtspraxis zur Verbesserung der Leseflüssigkeit eingesetzt werden (siehe 2.3). Das flüssige Lesen der kleinen Textpassagen der jeweiligen Rolle ist nur ein erster Schritt hin zum flüssigen Lesen eines längeren fiktionalen Erzähltextes. Mit der Zeit können aber die Umfänge der Passagen ausgeweitet werden. Weitere zentrale Förderdimensionen des Unterrichtsdesigns MELT sind einerseits die Lesemotivation und andererseits - anders als bei herkömmlichen monolingualen Lesetheatern - die Mehrsprachigkeitskompetenz von SchülerInnen (siehe 2.4 und 2.5).

\subsection{Leseflüssigkeit}

Leseflüssigkeit zählt zu den basalen Lesefertigkeiten, die die Voraussetzung für hierarchiehöhere Lesekompetenzen wie beispielsweise das sinnerfassende Lesen sind (vgl. Rosebrock et al. 2011: 15-20): Erst wenn LeserInnen in der Lage sind, einen Text flüssig zu lesen, stehen ihnen genügend kognitive Ressourcen zur Verfügung, um den Inhalt eines Textes zu rekonstruieren. Das Konzept Leseflüssigkeit setzt sich aus mehreren Teilfertigkeiten zusammen: der Lesegenauigkeit, der Automatisierung von basalen Dekodierprozessen, der Lesegeschwindigkeit sowie der sinngemäßen Betonung. Gute LeserInnen dekodieren Wörter im Vergleich zu weniger guten genauer, ihre Dekodierfähigkeit ist stärker automatisiert, sie lesen vergleichsweise schneller und sind in der Lage, syntaktische und semantische Beziehungen von sprachlichen Elementen innerhalb eines Textes zu erkennen und diese beim lauten Vorlesen durch ihren Stimmeinsatz (beispielsweise durch Pausen oder Betonungen) zu markieren (vgl. ebd.).

Dass sich der Einsatz von Lesetheatern positiv auf die Leseflüssigkeit in der Erstsprache auswirkt, konnte bereits mehrfach gezeigt werden (vgl. Mraz et al. 2013; Young \& Rasinski 2009; Clark et al. 2009; Martinez et al. 2002; Worthy \& Prater 2002; Tyler \& Chard 2000; speziell zur Leseförderung bei leistungsschwächeren SchülerInnen siehe z. B. Corcoran \& Davis 2005; Worthy \& Prater 2002). Zur Leseflüssigkeit in der L2 liegen bisher nur wenige gesicherte Erkenntnisse vor. Erste empirische Studien deuten jedoch darauf hin, dass erhöhte Leseflüssigkeit in der L2 ebenfalls mit besseren Verstehensleistungen in der L2 korreliert (vgl. Grabe 2014: 11). 


\subsection{Lesemotivation}

Lesemotivation ist neben der Leseflüssigkeit ein weiterer Ansatzpunkt zur Förderung von Lesekompetenz: Lesefreude und -motivation sind Faktoren, die die Lesehäufigkeit erhöhen können; die Lesehäufigkeit korreliert wiederum positiv mit Lesekompetenz (vgl. Cunningham \& Stanovich 1997). Das Unterrichtsdesign MELT wird dementsprechend so konzipiert, dass es möglichst lesemotivierend wirkt. Grundlage für die Entwicklung von motivationsfördernden Designelementen von MELT ist die Selbstbestimmungstheorie von Deci \& Ryan (2000). Der Theorie liegt die Annahme zugrunde, dass intrinsische Motivation stark mit den psychischen Grundbedürfnissen des Menschen nach Kompetenz, Autonomie und sozialer Eingebundenheit verbunden ist. Die intrinsische Motivation zur Ausführung einer Handlung wird von Bedingungen gefördert, die zur Befriedigung der drei Grundbedürfnisse beitragen; umgekehrt wird die intrinsische Motivation reduziert, sofern diese Bedingungen nicht gegeben sind (vgl. ebd. 233). Das Unterrichtsdesign MELT weist mehrere Charakteristika auf, die diesen drei Grundbedürfnissen entgegenkommen: Während der Vorbereitung auf die Aufführung handeln die SchülerInnen in unterschiedlichen Phasen autonom: Sie setzen sich selbstbestimmt mit ihrer Rolle auseinander, indem sie sich selbstständig die sprachliche Gestaltung der zu lesenden Rolle erarbeiten. Sie erleben sich darüber hinaus als kompetent, wenn sie ihre Rolle nach den Übungsphasen flüssig lesen können oder wenn sie aufgrund der Mehrsprachigkeit der Lesetheaterstücke in der Lage sind, Szenen aus Texten zu präsentieren, deren eigenständige Erarbeitung und Lektüre für sie ausschließlich in der Fremdsprache noch nicht möglich wäre. Und schließlich erleben sie sich als sozial eingebunden, wenn sie das Stück für ein Publikum präsentieren, dafür positives Feedback erhalten und sich angenommen fühlen.

\subsection{Mehrsprachigkeit}

In MELT werden literarische Texte aus der Kinder- und Jugendliteratur in mehrsprachige dialogische Lesetheaterstücke umgewandelt. Je nach landesspezifischem Praxiskontext werden verschiedene Sprachkombinationen aus Schul-/Fremd- und Migrationssprache in den Texten verwendet. Der Einsatz von mehrsprachigen Texten im Unterricht hat laut Eder (2015: 148-170) unterschiedliche Funktionen: „(sprachen)politische“, „(sprachen)didaktische“ und „formal-ästhetische“. Insbesondere die ersten beiden Funktionen sind für MELTTexte relevant: Zum einen kommt MELT-Texten eine sprachenpolitischeFunktion zu, indem sie Mehrsprachigkeit sichtbar machen und die Gleichwertigkeit der in den Texten verwendeten Sprachen hervorheben. Durch die gleichberechtigte Verwendung von Sprachen wird insbesondere auch der Status von so genannten Minderheitensprachen erhöht (vgl. ebd. 148f.). Zum anderen kommen den mehrsprachigen MELT-Lesetexten auch sprachdidaktische Funktionen zu, die in diesem Beitrag allerdings nur skizziert werden können: Das Unterrichtsdesign sieht vor, dass sich die SchülerInnen die Inhalte der mehrsprachigen Lesetexte in Gruppen eigenständig erarbeiten. Die mehrsprachige Gestaltung der Texte 
regt idealerweise sprachübergreifende Rezeptionsprozesse an, beispielsweise indem fremdsprachige Passagen unter Rückgriff auf die deutschsprachigen Stellen entschlüsselt werden. Die SchülerInnen werden so im Einklang mit neuen Ansätzen in der Mehrsprachigkeitsdidaktik dazu angeregt, das gesamte sprachliche Repertoire, das ihnen in der Situation zur Verfügung steht, zur Bewältigung der Aufgabe zu nutzen. Diese Form des sprachübergreifenden Arbeitens unterstützt implizit den Auf- und Ausbau von „metalinguistic“ und „crosslinguistic awareness“ (vgl. Allgäuer-Hackl \& Jessner 2013: 125). Die in MELT zu beobachtenden sprachübergreifenden Rezeptionsprozesse werden im Zuge der Erprobungsphasen im Detail analysiert und beschrieben werden.

\section{Entwicklung und Umsetzung von MELT-Design-Elementen in der Praxis}

\subsection{Auswahl von Texten und Entwicklung von Lesetheaterstücken}

Für die Auswahl der Lesetheatertexte spielen verschiedene Kriterien eine Rolle. Im MELT-Unterricht sollen die SchülerInnen die Gelegenheit haben, Werke der erzählenden Kinder- und Jugendliteratur aus verschiedenen europäischen Sprachräumen (konkret aus dem anglo-, franko- und dem germanophonen Sprachraum) kennenzulernen. Darüber hinaus sollen sie sowohl mit verschiedenen Genres, Klassikern und bekannten Bücherserien als auch mit internationalen Bestsellern der Kinder- und Jugendliteratur vertraut werden. $\mathrm{Zu}$ den weiteren Kriterien zählt die Auseinandersetzung mit Texten, die ein hohes Identifikationspotenzial für Mädchen und Jungen aufweisen, die reich an Dialogen sind und sich für die ausgewählte Altersstufe eignen. Bisher wurden folgende Lesetheatertexte in Klassenstufe 5 und 6 (Schweiz) bzw. 7 und 8 (Deutschland, Österreich, Luxemburg) je nach Land in verschiedenen Sprachkombinationen (Deutsch, Englisch, Französisch, Migrationssprachen) erarbeitet: Die Kleinen Wilden, Die Wilden Fußballkerle, Billy Elliot, Bend it like Beckham, The Horse Whisperer, Moondial, Krabat, Tom Sawyer, Sherlock Holmes, Le Petit Nicolas, Harry Potter Band 1, Der Kleine Werwolf, The Tales of Nasreddin Hodscha und The Canterville Ghost.

Auf die verschiedenen Versionen der Lesetheaterstücke, die sich aus der Überarbeitung der Texte aufgrund des DBR-Ansatzes ergeben, kann in diesem Beitrag aus Platzgründen nicht eingegangen werden. Im Folgenden wird daher von einer bestimmen Version des Lesetheaterstückes ausgegangen.

\subsection{Mehrsprachige Dramatisierung von Prosatexten}

Die Prosatexte werden in einem weiteren Schritt mehrsprachig dramatisiert. Der folgende kurze Ausschnitt aus dem Lesetheaterskript The Tales of Nasreddin Hodscha, der in Deutschland eingesetzt wurde, veranschaulicht exemplarisch die Verwendung verschiedener Sprachen innerhalb der Lesetheatertexte. Die beiden Erzählerrollen sind in der Schulsprache Deutsch geschrieben, die 
Charaktere hingegen in englischer Sprache, teilweise auch mit Anteilen in der Migrationssprache Türkisch (dieser Text wurde von Miriam Häberle im Rahmen einer Qualifikationsarbeit an der PH Weingarten erstellt).

\begin{tabular}{|l|l|}
\hline Narrator 1 & $\begin{array}{l}\text { Eines Tages ging Nasreddin Hodscha mit seinem Sohn auf einen Viehmarkt, } \\
\text { um dort einen Esel zu kaufen. }\end{array}$ \\
\hline Narrator 2 & $\begin{array}{l}\text { Nach langer Suche kauften sie en dlich einen Esel und machten sich mit ihm } \\
\text { auf den Weg nach Hause. }\end{array}$ \\
\hline Narrator 1 & $\begin{array}{l}\text { So liefen die beiden zu Fuß den steinigen Weg entlang, während der Esel } \\
\text { nebenher trottete. Bis ihnen ein Wanderer begegnete... }\end{array}$ \\
\hline Hiker & Hahaha, you guys are weird. You have a donkey, but no one rides on it! \\
\hline Nasreddin & I think he is right, my son. You should ride the donkey, I will walk alongside. \\
\hline Nasreddin's son & Tamam, babam(Ok, dad). \\
\hline
\end{tabular}

Pro Lesetheaterskript kommen lediglich drei bis maximal vier unterschiedliche Sprachen vor. Jedes am Projekt beteiligte Land entscheidet ausgehend von der länderspezifischen Situation, welche Sprachenkombinationen berücksichtigt werden. Neben der jeweiligen Schulsprache sind dies die Fremdsprachen, die in diesen Ländern hauptsächlich gelernt werden, sowie die Migrationssprachen, die in den beteiligten Klassen von den Jugendlichen am häufigsten gesprochen werden. Bei der Übersetzung der Passagen in die Migrationssprachen werden die einzelnen Projektteams von kompetenten SprecherInnen der in den Lesetheatern verwendeten Sprachen unterstützt.

\subsection{MELT-Prozessschritte}

Nachfolgend wird kurz skizziert, welche Prozessschritte für die kooperierenden Lehrpersonen relevant sind, um Lesetheater praktikabel und erfolgreich im Unterricht einzusetzen. Die Prozessschritte umfassen zentrale Lehr- und Lernprozesse: vom ersten Vorlesen des Textes durch die Lehrkraft und Situierung der Lektüre bis hin zur Aufführung des Lesetheaters mit möglicher anschließender Reflexion. Die unten angeführten Prozessschritte wurden auf der Grundlage einer vorausgegangenen Interventionsstudie ausgearbeitet (vgl. Villiger et al. 2010), in der Lesetheater als Lesefördermaßnahme eine zentrale Rolle spielte. Zudem lehnen sich die Lehr- und Lernprozessschritte an die didaktisch-methodischen Prinzipien der Fremdsprachen- und der Schulsprachendidaktik an. Nachdem den Kooperationslehrkräften ein erster Entwurf vorgelegt worden war, flossen diese Rückmeldungen in eine erste Überarbeitung ein. Es kristallisierten sich folglich acht relevante Lehr- und Lernprozessschritte heraus, die gemäß den schulischen Gegebenheiten von den kooperierenden Lehrpersonen auf ungefähr zwei bis drei Tage innerhalb einer Schulwoche verteilt wurden und die mit verschiedenen Sozialformen (SF) verknüpft wurden: Plenum (PL), EA (Einzelarbeit), GA (Gruppenarbeit). 
- Einführung des Lesetheaters (PL): Das Lesetheater, das die SchülerInnen später in Arbeitsgruppen weitgehend selbständig lesen und bearbeiten, wird durch die Hinführung zum Thema und eine Vorstellung der Charaktere vorentlastet. Gegebenenfalls werden auch die fremdsprachlichen Voraussetzungen geschaffen, um das Verständnis zu erleichtern. Dramapädagogische Aktivitäten wie Erzählübungen, wie sie im Abschnitt 4.1. beschrieben werden, stimmen die Lernenden auf die Geschichte ein (oder auch als 2. Schritt)

- Vorlesen der Lehrperson (PL): Die Lehrperson liest eine zentrale Passage aus der literarischen Vorlage mehrsprachig vor. Somit erleben die SchülerInnen ihre Lehrerin/ihren Lehrer als Modell für die hör- und sichtbaren Mittel der Textgestaltung (oder auch als 1. Schritt)

- Lesen der Lesetheaterszene und Rollenverteilung (EA/GA): Die SchülerInnen teilen sich in Arbeitsgruppen auf. Jede/jeder SchülerIn liest ihre/seine Lesetheaterszene und verschafft sich einen inhaltlichen Überblick über die Szene. Im Anschluss werden die Leserollen verteilt und wichtige Zusammenhänge für das Verständnis der Lesetheaterszene geklärt.

- Erarbeiten einer Inhaltsangabe der Lesetheaterszene (GA): Die SchülerInnen lesen in verteilten Rollen ihre Szene ein zweites Mal und klären miteinander Satzzusammenhänge (lokale Kohärenzbildung) sowie auftretende Verständnisprobleme bei Schlüsselbegriffen, insbesondere in den Fremd-/Migrationssprachen. Die mehrsprachigen Texte sind häufig so gestaltet, dass unbekannte Wörter oder Passagen durch Rückgriff auf schulsprachliche Teile erschlossen werden können. Die SchülerInnen erarbeiten im Anschluss eine mündliche Zusammenfassung ihrer Szene, die den Inhalt kurz und übersichtlich wiedergibt. Dadurch klären die SchülerInnen für das Verständnis der Lesetheaterszene als Ganzes wichtige Zusammenhänge (globale Kohärenz). Leitfragen der Lehrperson unterstützen die Lernenden in diesem Prozess.

- Vorstellen aller Lesetheaterszenen und Auseinandersetzung mit einzelnen Leserollen (PL): Damit die SchülerInnen ihre Leserolle im Anschluss selbstständig erarbeiten können, benötigen sie eine Übersicht über den Inhalt des gesamten Lesetheaters. Jede Arbeitsgruppe stellt daher ihre Lesetheaterszene im Klassenverband vor. Um das Textverständnis zu vertiefen, bieten sich in dieser Phase dramapädagogische Aktivitäten an, wie sie im Abschnitt 4.1 beschrieben werden. In dieser Phase der Textarbeit können auch Gespräche zur literarischen-ästhetisch Auseinandersetzung mit Texten geführt werden

- Üben der Leserollen und gegenseitiges Feedback (EA/GA): Die SchülerInnen lesen ihre Szene mehrmals halblaut und geben sich Rückmeldungen. Während der ersten Übungsdurchgänge stehen insbesondere bei 
leseungeübten SchülerInnen das korrekte Dekodieren und die korrekte Aussprache im Vordergrund. Mit zunehmender Automatisierung auf Wortund Satzebene stehen den SchülerInnen mehr kognitive Ressourcen zur Verfügung, um den Text vertieft zu analysieren. Ein gutes Verständnis der Szene verbessert die prosodische Lesegestaltung, die SchülerInnen konzentrieren sich dann beim Üben ihrer Leserolle vermehrt auf das Gliedern in Sinnabschnitte, auf den Ausdruck, die Akzentuierung sowie die Intonation und Pausensetzung.

- Generalprobe (GA): Jede Arbeitsgruppe führt eine Generalprobe ihrer Lesetheaterszene durch, bei der die einzelnen Leserollen aufeinander abgestimmt und im Zusammenspiel geübt werden. Dies bietet den Lernenden die Gelegenheit, die Wirkung der Vorlesegestaltung zu reflektieren und zu prüfen, ob die Vorführung für ein Publikum unterhaltsam ist. Während der Generalprobe sind Rückmeldungen von der Lehrperson und/oder anderen SchülerInnen hilfreich, damit die Arbeitsgruppe die Wirksamkeit der sichtbaren Mittel der Vorlesegestaltung (Blickkontakt, Gestik und Mimik) einschätzen lernt.

- Aufführung (PL): Jede Arbeitsgruppe liest ihre Lesetheaterszene im Klassenverband vor. Für die SchülerInnen, die zusehen und zuhören, soll die Aufführung des gesamten Lesetheaters unterhaltsam sein. Als Vorbereitung auf die Aufführung können dramapädagogische Übungen eingesetzt werden, die die performativen Kompetenzen der SchülerInnen stärken (siehe Abschnitt 4.2). Der Applaus am Ende des Auftritts ist wie im richtigen Theater der Lohn für die Anstrengungen und die gezeigten Lernfortschritte beim gestaltenden Lesen von mehrsprachigen Lesetheatern.

\section{Dramapädagogische Übungen im Kontext von Lesetheater}

Der Einsatz dramapädagogischer Methoden in MELT kann einerseits eine Hilfe bei der Durchführung der einzelnen Prozessschritte sein, andererseits stellt er grundsätzlich eine wertvolle Bereicherung des Designs dar: Durch die Einbindung von Elementen aus dem Bereich des Theaters werden kommunikative und sprachliche sowie soziale und performative Kompetenzen der Lernenden gestärkt. Im Rahmen von MELT können kreative, ganzheitlich orientierte Methoden aus der Dramapädagogik im Sprachenunterricht stark motivierend wirken und auf diese Weise zum Lernerfolg beitragen.

Im Folgenden sollen die Funktionen von dramapädagogischen Übungen bei der Umsetzung in MELT jeweils mithilfe konkreter Umsetzungsmöglichkeiten illustriert werden. Die Entscheidung, ob und an welcher Stelle theaterpädagogische Methoden zum Einsatz kommen, obliegt der Lehrperson, da diese Elemente als mögliche Erweiterung des Designs betrachtet werden können. 
Die Literatur zur allgemeinen theaterpädagogischen Praxis bietet eine Fülle von praktischen Anregungen (z.B. Vlcek 2006 oder Brem 2005), die sich teilweise auch in der Schule umsetzen lassen. In englischsprachigen Veröffentlichungen finden sich Beschreibungen zahlreicher bereits bewährter dramapädagogischer Methoden im Fremdsprachenunterricht (Maley \& Duff 1978/2005, Swale 2009). Die in diesem Beitrag genannten Beispiele können nur einen kleinen Teil der vorhandenen Möglichkeiten widerspiegeln. Der Großteil der hier für MELT vorgestellten Übungen bezieht sich auf das in MELT entwickelte mehrsprachige Lesetheater Nasreddin Hodscha; gelegentlich wird auch auf andere Lesetheater, die im Rahmen von MELT erarbeitet wurden, Bezug genommen (siehe Abschnitt 3.1). Die Handlung des Lesetheaters Nasreddin Hodscha ist rasch beschrieben: In der ersten Szene hat Nasreddin Hodscha gemeinsam mit seinem Sohn am Markt einen Esel gekauft. Beeinflusst durch Kommentare von Wanderern, denen sie begegnen, reitet zuerst der Sohn, dann der Vater, dann beide gemeinsam, bis sie am Ende selbst den Esel nach Hause tragen! Das Stück soll auf humorvolle Weise verdeutlichen, was passiert, wenn man versucht, es allen recht zu machen.

In den Beschreibungen werden die folgenden Abkürzungen verwendet: Lehrperson $=$ LP, Schülerinnen und Schüler $=$ SuS, Lesetheater $=$ LT. Die Ideen zur konkreten Umsetzung der Übungen „Freie Dialoge“ und "Vortragswettbewerbe“ in MELT stammen vom MELT-Kooperationslehrer Kurt Benedikt.

\subsection{Dramapädagogische Elemente als Unterstützung bei der Durchführung von MELT-Prozessschritten}

Dramapädagogische Elemente können in verschiedenen Phasen des MELTUnterrichtsdesigns eingesetzt werden und erfüllen mehrere Funktionen. $\mathrm{Zu}$ diesen zählen die Vorbereitung auf das Lesetheater, die Unterstützung der Lernenden bei der Erarbeitung der mehrsprachigen Lesetheaterstücke, bei der Erschließung des Inhaltes und der Entwicklung der Figuren, Hilfestellung in den Übungsphasen und bei der Vorbereitung auf die Generalprobe und die Aufführung.

Vorbereitung auf MELT-Lernprozesse - Dramapädagogische Übungen sind geeignet, die Lernenden körperlich und mental auf den Lernprozess vorzubereiten (vgl. Bonnet \& Küppers 2011: 50) und thematisch zur Geschichte und zum Stoff hinzuführen (vgl. Haack 2011: 62). Die Wirkung solcher Aufwärmübungen reicht von der Entspannung und der Gruppenbildung bis hin zur Konzentration und "Schulung der individuellen sinnlichen Wahrnehmungsfähigkeit" (Czerny 2004: 168). Eine wichtige Funktion erfüllen in dieser Phase auch stimmliche Übungen (vgl. Maley \& Duff 1978/2005: 69-98) oder Methoden, die der Verbesserung der nonverbalen Kommunikation dienen. Aufwärmübungen sollten keineswegs nur als Spielerei betrachtet werden: Bezugnehmend auf verschiedene Studien (Kempe \& Nicholson 2007; Neelands 2010; Winston \& Tandy 1998) 
argumentiert Kempston (2012: 95), dass die Teilnahme an Aufwärmübungen als ,a necessary pre-requisite for successful drama“ anzusehen sei.

\section{Aufwärmübung - Bewegungsübung}

\begin{tabular}{|l|l|}
\hline Ziel & $\begin{array}{l}\text { Stärkung des Körperbewusstseins, Hinführen zum Thema des Lesetheaters. Die } \\
\text { konkrete Übung eignet sich thematisch gut als Aufwärmübung für das Lesetheater } \\
\text { Nasreddin Hodscha. }\end{array}$ \\
\hline Quelle & Ähnliche Übungen werden von Haack \& Surkamp (2011: 62) beschrieben. \\
\hline
\end{tabular}

Die SuS sollen sich durch den gesamten Raum bewegen, zuerst so, als ob sie eine schwere Last tragen. Nach kurzer Zeit gibt die LP die Anweisung, dass sich die SuS nun so bewegen sollen, als ob sie selbst federleicht wären oder schweben könnten. Diese Übung kann je nach Leistungsstand der Lernenden auch zweisprachig oder mehrsprachig durchgeführt werden, wodurch die SuS bereits auf das mehrsprachige Setting vorbereitet werden.

Erzählübung - Objekte erzählen lassen

\begin{tabular}{|l|l|}
\hline Ziel & $\begin{array}{l}\text { Freies Erzählen üben als Vorbereitung auf das Sprechen und Agieren in einer } \\
\text { Rolle. }\end{array}$ \\
\hline Quelle & $\begin{array}{l}\text { Beschreibungen ähnlicher Übungen finden sich bei Schäfer (2006: 20) und Swale } \\
(2015: 126) .\end{array}$ \\
\hline
\end{tabular}

Die LP bringt zwei Gegenstände mit, die zum Thema des Lesetheaters passen. Ein/e Schüler/in, der/die Lust hat, mit dem Erzählen zu beginnen, darf eines der zwei Objekte auswählen. Aufgabe ist es nun, eine Geschichte zu erzählen, in der der Gegenstand vorkommt, wobei dieser nicht gleich zu Beginn der Erzählung erwähnt werden darf. Eine andere Möglichkeit ist es, die Geschichte aus der Sicht des Gegenstandes erzählen zu lassen.

Erzählübung - Ein-Satz-Geschichte

\begin{tabular}{|l|l|}
\hline Ziel & $\begin{array}{l}\text { Konzentration, Stärkung der Vorstellungskraft, echtes Zuhören, deutliches } \\
\text { Sprechen, Hinführung zum Thema des LT. }\end{array}$ \\
\hline Quelle & $\begin{array}{l}\text { Verschiedenste dramapädagogische Übungen mit Fokus auf dem Erzählen werden } \\
\text { von Martens (2008), Wardetzky (2007), Steinweg (2006), Brem (2005) und Walk } \\
\text { (2015) beschrieben. }\end{array}$ \\
\hline
\end{tabular}

Die LP formuliert die ersten Worte einer Geschichte, die zum Thema des Lesetheaters passt. Die SuS sollen nacheinander jeweils ein Wort ergänzen und die Geschichte weiterführen; die Worte des Vorgängers werden jeweils wiederholt. Zum Lesetheater Nasreddin Hodscha könnte passen: „Ich hatte einmal einen Esel, der ... “ oder „Gestern ritt ich ... “ oder „Mein Vater und ich..." 
Dramapädagogische Übungen dieser Art, in denen der Fokus auf dem Erzählen liegt, spiegeln das gegenwärtig starke Interesse auf dem Gebiet der Dramapädagogik am dramatischen und kreativen Prozess des Erzählens wider.

Erschließen von MELT-Lesetexten - Eine wichtige Funktion kommt der Dramapädagogik im Projekt MELT bei der Erschließung des Inhalts und der Herausarbeitung der Figuren zu, da die erfolgreiche Bewältigung dieser Aufgaben Voraussetzungen für das gestaltende Lesen und das Übernehmen von Rollen darstellen. Durch die ganzheitliche Orientierung dieser Lernmethode bieten die Übungsformen der Dramapädagogik den SuS vielfältige Anknüpfungspunkte beim Erfassen des Inhalts. Im Hinblick auf den Umstand, „,[that] young learners of a foreign language need more visual and aural assistance in order to understand a text" (Chang 2012: 10), scheinen dramapädagogische Übungen geeignet, gute Bedingungen für das Lesen mehrsprachiger Texte in Klassen jüngerer SchülerInnen zu schaffen.

\section{Standbildtechnik - Freeze Frame-Technik}

\begin{tabular}{|l|l|}
\hline Ziel & $\begin{array}{l}\text { Bewusstwerden von kritischen Momenten der Handlung, aktive } \\
\text { Auseinandersetzung mit der Gedanken- und Gefühlswelt der Figuren; die } \\
\text { Standbildtechnik kann auch als Effekt auf der Bühne eingesetzt werden. }\end{array}$ \\
\hline Quelle & $\begin{array}{l}\text { Die Standbildtechnik zählt zu den etablierten Methoden der Dramapädagogik. } \\
\text { Beschreibungen finden sich bei u.a. bei Even (2011: 75), Haack \& Surkamp (2011: } \\
\text { 62), Kessler (2008: 126), Brem (2005: 62). }\end{array}$ \\
\hline
\end{tabular}

Die SuS erhalten bei dieser Methode den Auftrag, einen oder mehrere Schlüsselmomente ihrer Leseszenen als eingefrorene Momente körperlich darzustellen. Im Rahmen der Unterrichtserprobung des Lesetheaters Billy Elliot wurde den SuS nach der Lektüre ihrer Leseszenen die Aufgabe gestellt, sich eine typische Bewegung und eine Haltung ihrer Figur zu überlegen. In einem weiteren Schritt wurden die Figuren gebeten, mit ihrer Bewegung mit anderen Figuren in Interaktion zu treten. Sobald die LP „Freeze“ oder „Standbild“ rief, erstarrten die SuS in ihrer Bewegung und behielten diese Position für kurze Zeit bei. Im Anschluss daran wurden die SuS von der LP aufgefordert, ihr Standbild zu erklären und sprachlich auszudrücken, was die Figur im Moment der Darstellung empfindet. Um das Verständnis der einzelnen Rollen sicherzustellen, wurden die SuS auch gebeten zu erläutern, in welcher Beziehung ihre eigene Figur zu den anderen Figuren in der Lesetheaterszene steht.

Auseinandersetzung und Identifikation mit MELT-Leserollen — Ein charakteristischer Bestandteil theaterpädagogischer Arbeit ist das spielerische Übernehmen von Rollen und das Experimentieren mit anderen Identitäten. Im Projekt „Mehrsprachiges Lesetheaterërweist sich gerade dieser Aspekt als besonders wertvoll: Er ermöglicht es den Lernenden, sich mit der Gefühls- und Gedankenwelt der 
Charaktere, denen sie in den Leseszenen begegnen, persönlich auseinanderzusetzen und diese Figuren mit Charaktereigenschaften, Wünschen und Motiven in ihrer eigenen Vorstellungswelt entstehen zu lassen. Bei dramapädagogischen Übungen, wie etwa bei der Entwicklung von Standbildern oder Methoden wie „Thought Tapping“, wird die intensive Beschäftigung mit den Charakteren als kreativer Prozess erlebt.

\section{Thought Tapping}

\begin{tabular}{|l|l|}
\hline Ziel & Intensive Auseinandersetzung mit den Figuren. \\
\hline Quelle & $\begin{array}{l}\text { „Thought Tapping“ zählt zu den gängigen dramapädagogischen Methoden. Eine } \\
\text { Beschreibung der Übungsform findet sich u.a. bei Bonnet \& Küppers (2011). }\end{array}$ \\
\hline
\end{tabular}

Bei dieser Übung werden Gedanken und emotionale Zustände hörbar gemacht, indem einzelne SuS ihre Hand auf die Schulter einer „eingefrorenen Figur" legen und dabei deren Gedanken formulieren (vgl. Bonnet \& Küppers 2011: 44). Die Äußerungen können entweder in der Muttersprache oder auch in einer der Fremdsprachen erfolgen. Zur vertieften Auseinandersetzung mit den Figuren können die in der Übung hörbar gewordenen Gedanken anschließend im Plenum reflektiert werden. Die Übung „Thought Tapping“ lässt sich gut mit der Standbildtechnik verknüpfen.

\section{Hot Seating}

\begin{tabular}{|l|l|}
\hline Ziel & Auseinandersetzung mit der Gefühls- und Gedankenwelt der Figuren. \\
\hline Quelle & $\begin{array}{l}\text { „Hot Seating“ gehört zum traditionellen Übungsrepertoire der Dramapädagogik. } \\
\text { Beschreibungen dieser Übungsform finden sich u.a. bei Kessler (2008: 127). }\end{array}$ \\
\hline
\end{tabular}

Bei dieser Methode muss die Figur, die sich auf dem „heißen Stuhl“ befindet, dem Plenum Rede und Antwort stehen. Das Sitzen auf dem Stuhl ist in dieser Übung das Zeichen für die Rollenübernahme. Beim Lesetheater Nasreddin Hodscha könnten beispielsweise Nasreddin oder sein Sohn von der Dorfgemeinschaft oder seiner Familie befragt werden.

\subsection{Dramapädagogische Elemente zur Stärkung der von MELT geförderten Kompetenzen}

Performative Kompetenzen in der Schul- und Fremdsprache - Performative Kompetenzen kommen im Projekt MELT vor allem bei der Generalprobe und bei der Aufführung zum Tragen. Durch die Einbindung von Mitteln aus dem Bereich des Theaters und der schauspielerischen Ausbildung können SchülerInnen lernen, wie ihre Körpersprache wirkt und wie sie Präsenz auf der Bühne erzeugen. In stimmlichen Übungen werden unter anderem Ausdruck und Intonation sowie Klarheit beim Sprechen geschult. 


\section{Freie Dialoge ${ }^{1}$}

\begin{tabular}{|l|l|}
\hline Ziel & Freies Sprechen üben. \\
\hline Quelle & $\begin{array}{l}\text { Verschiedenste Übungen zum freien Experimentieren mit Texten finden sich bei } \\
\text { Hilliger (2009). Idee zur Umsetzung in MELT: Kurt Benedikt (2015). }\end{array}$ \\
\hline
\end{tabular}

Die SuS sind angehalten, sich frei in der Klasse zu bewegen. Wenn sie jemandem begegnen, zitieren sie einen beliebigen Satz aus ihrer Rolle. Die Person, der sie begegnen, antwortet mit einem beliebigen Satz ihrer Rolle.

Vortragswettbewerbe ${ }^{2}$

\begin{tabular}{|l|l|}
\hline Ziel & $\begin{array}{l}\text { Freies Sprechen üben, Lautlesetraining, Vorbereitung auf die Generalprobe und } \\
\text { auf die Aufführung. }\end{array}$ \\
\hline Quelle & Idee zur konkreten Umsetzung in MELT: Kurt Benedikt (2015). \\
\hline
\end{tabular}

Die SuS erhalten vorbereitend alle Sätze ihrer Leseszene, die in der/den Fremdsprache/n gesprochen werden, auf einem separaten Blatt. Im Rahmen eines kleinen Wettbewerbs tragen nun alle SuS einer Arbeitsgruppe nacheinander jeweils einen dieser Sätze vor, wobei die Sätze vom Blatt gelesen werden dürfen. Zwei andere SuS fungieren als Jury und bewerten die Sätze im Hinblick auf folgende Kriterien: mutiges Auftreten, Deutlichkeit beim Sprechen, korrekte Aussprache (mit Blick auf die Fremdsprache), Ausdruck und Intonation. Für jedes erfüllte Kriterium erhält die Gruppe einen Punkt oder Stempel. Bei der Bewertung der Aussprache in der Fremdsprache kann die LP gegebenenfalls helfen.

Übungen nach der Aufführung - Inszenierung einer Fortsetzungsgeschichte

\begin{tabular}{|l|l|}
\hline Ziel & Intensivere Auseinandersetzung mit der Geschichte und den Figuren. \\
\hline Quelle & $\begin{array}{l}\text { Zum kreativen Arbeiten mit Texten und zum Prinzip der Texterweiterung } \\
\left(, \text { expansion }{ }^{(2)} \text { siehe Maley (1995).| }\right.\end{array}$ \\
\hline
\end{tabular}

Die SuS sollen in Gruppen eine kleine Szene erarbeiten, aus der hervorgeht, was sich nach dem Ende der Geschichte ereignet haben könnte. Die Szenen werden niedergeschrieben, kurz geübt und vorgetragen. Im Fall des LT Nasreddin Hodscha könnten die SuS beispielsweise einen Dialog gestalten über das Gespräch, das Nasreddin nach seiner Rückkehr mit seiner Frau führt, oder eine Szene zum Geschwätz der Dorfbewohner über die seltsame Begebenheit.

Erhöhung der Sprachlern- und Lesemotivation - Die spielerische Art und Weise dramapädagogischer Methoden kann bei der Arbeit am Text und an den Figuren

\footnotetext{
${ }^{1}$ Es handelt sich hierbei um unveröffentlichte Unterrichtsmaterialien.

${ }^{2}$ Siehe Fußnote 1.
} 
stark motivierend wirken (vgl. Schmidt 2008: 35), da die Lernenden nicht auf die Rolle von Rezipienten von Texten beschränkt bleiben, sondern zu Akteuren werden, die bei den Übungen gemeinsam Verantwortung tragen und den Unterricht aktiv mitgestalten.

In den Übungsphasen zur Vorbereitung auf die Generalprobe bieten dramapädagogische Mittel die Möglichkeit, das notwendige Sprech- und Lesetraining, das viele Wiederholungen miteinschließt, motivierender und effektiver zu gestalten. Wer bereits mit dramapädagogischen Methoden gearbeitet hat, weiß: In einer Atmosphäre des Vertrauens haben junge Menschen Freude daran, in andere Rollen zu schlüpfen. Diese spielerische Energie kann genutzt werden, um die verschiedenen Aufgaben, die SchülerInnen im Rahmen des Lesetrainings meistern müssen, zu erleichtern. Durch die Verknüpfung der Lesetheaterszenen mit dramapädagogischen Übungen erleben SchülerInnen das Lesen und die Beschäftigung mit literarischen Texten im Unterricht als ereignishaften, spannenden und oft vergnüglichen Prozess (vgl. Schewe 2011: 26). Diese positive Erfahrung motiviert zur weiteren Lektüre und zur individuellen Auseinandersetzung mit den literarischen Texten, auf denen die Lesetheaterstücke beruhen.

Die zentrale Bedeutung motivationsfördernder Elemente in MELT - einem Design, das u.a. auf der Grundlage der Selbstbestimmungstheorie von Deci \& Ryan (2000) konzipiert wurde - wurde bereits im Abschnitt 2.4. dargelegt. Dramapädagogische Übungen schaffen Lernbedingungen, in denen sich die SchülerInnen einerseits als sozial eingebunden und andererseits als kompetent erleben können, was sich beides wiederum positiv auf die intrinsische Motivation, sich mit dem Lerngegenstand auseinanderzusetzen, auswirken kann.

\section{Soziale Eingebundenheit}

Zentrale Prinzipien dramapädagogischer Übungen sind die gegenseitige Unterstützung der teilnehmenden SchülerInnen, Respekt und Vertrauen sowie die Bereitschaft, zusammenzuarbeiten. Ein wertvoller Aspekt dramapädagogischer Übungen besteht daher darin, dass sich die SchülerInnen beim Lernen mit dramapädagogischen Methoden verstärkt sozial eingebunden und angenommen fühlen können. Auf diese Weise unterstützen dramapädagogische Übungen ein zentrales Prinzip von MELT. Charakteristisch für theaterpädagogische Übungen ist auch die Erfahrung der SchülerInnen, dass für den Erfolg des Ganzen das Zusammenspiel der Gruppe ebenso wichtig ist wie die individuelle Leistung des Einzelnen. Durch dramapädagogische Übungen werden SchülerInnen mit kooperativen Lernformen vertraut und ihre sozialen Kompetenzen werden gestärkt.

\section{Kompetenzerleben}

Eine weitere wichtige Funktion dramapädagogischer Übungen im Fremdsprachenunterricht ist die „Verbesserung von kommunikativen Kompetenzen“ (Bonnet \& Küppers 2011: 41). Der Erfolg dramapädagogischer Methoden, der bei der Verwirklichung dieses Ziels beobachtet wird, hängt damit zusammen, dass mithilfe theaterpädagogischer Methoden Situationen geschaffen werden, 
die die teilnehmenden SchülerInnen persönlich betreffen und in denen Kommunikation eine echte Notwendigkeit darstellt. Wenngleich diese Situationen fiktional sind, werden sie von den Lernenden im Moment gleichsam als real erlebt.

Aufgrund ihrer kommunikativen Ausrichtung finden dramapädagogische Übungen „Vor allem Eingang in den mündlichen Sprachunterricht“ (Fratini 2008: 45-46). Interessant ist aber auch die Frage, welchen Beitrag Dramapädagogik im Projekt MELT im Bereich der mündlichen fremdsprachlichen Kompetenz - auf der Ebene des Wortschatzes, beim Aufbau sprachlicher Strukturen und im Hinblick auf die Aussprache - zu leisten vermag. Erste Erprobungen im Unterricht mit MELT-Lesetheatern deuten darauf hin, dass SchülerInnen durch den Einsatz dramapädagogischer Mittel die Scheu vor dem Umgang mit der Fremdsprache oder der Zweitsprache verlieren und eher bereit sind, sich in der Fremd- oder Zweitsprache auszudrücken. Die Möglichkeit, die theaterpädagogische Übungen den Lernenden bieten, in Rollen zu schlüpfen und mit verschiedenen Identitäten zu experimentieren, erfüllt in diesem $\mathrm{Zu}$ sammenhang eine wichtige Funktion „enabling them to feel safe enough to take risks with language that they would otherwise feel too self conscious to attempt" (Winston 2012: 3). Darüber hinaus können für das Lesetheaterstück relevante Wörter und sprachliche Strukturen im Rahmen dramapädagogischer Übungen vorbereitet und eingeführt oder nach den Lesephasen wiederholt und gefestigt werden. Dass SchülerInnen im Rahmen dramapädagogischer Übungen häufig anderen zuhören, ist ein weiterer Aspekt, der sich im Kontext des Fremdsprachenerwerbs, etwa im Bereich des Wortschatzes, als wertvoll erweist, „reinforcing memorisation and understanding of the content" (Chang \& Winston 2012: 20). „Watching others act out the story enhances the learners' motivation and provides a meaningful context for the vocabulary taught" (ebd.); laut Maley \& Duff (1978/2005: 4) eignen sich dramapädagogische Übungen insbesondere zur Festigung von bereits bekannten sprachlichen Strukturen. Erste praktische Erfahrungen mit MELT - unter Einbeziehung dramapädagogischer Mittel - legen nahe, dass im Bereich der Aussprache relativ rasch Fortschritte erzielt werden können. Den für MELT relevanten Zusammenhang zwischen Verbesserungen im Bereich mündlicher Sprachkompetenzen und der Entwicklung von Kompetenzen im Bereich des Lesens und Schreibens stellt Chang her, wenn sie schreibt: „Drama is a key way to maximise opportunities for pupils' oracy, thus paving the way for developing literacy" (Chang 2012: 8). Ein für den Lernerfolg wichtiger Faktor ist dabei das Niveau der Texte. In ersten Erprobungen von MELT hat sich gezeigt, dass die mehrsprachigen Lesetheater relativ einfach sein müssen, damit die SchülerInnen sich in der Auseinandersetzung mit diesen Texten selbst als kompetent erleben können.

\section{Schlussfolgerungen und Ausblick}

Wie sich gezeigt hat, stellen dramapädagogische Verfahren aus mehreren Gründen eine wertvolle Ergänzung zur hier vorgestellten mehrsprachigen 
Lesefördermaßnahme „Mehrsprachiges Lesetheater“ dar: Sie können einerseits unterstützende Funktion bei der Durchführung der einzelnen in MELT vorgesehenen Prozessschritte übernehmen, andererseits können sie unmittelbar dazu beitragen, jene Kompetenzen zu fördern, auf die MELT abhebt. Vor diesem Hintergrund könnten sich Untersuchungen zur Wirksamkeit von dramapädagogischen Übungen zur Förderung von Leseflüssigkeit und Lesemotivation in fremdsprachlichen Lernumgebungen als lohnend erweisen. Der Unterrichtspraxis wäre zu wünschen, dass Lehrpersonen das hohe Potenzial von dramapädagogischen Elementen in Lesetheatern erkennen und in ihrem Unterricht vermehrt damit arbeiten.

\section{Bibliografie}

Allgäuer-Hackl, Elisabeth \& Jessner, Ulrike (2013):

Mehrsprachigkeitsunterricht aus mehrsprachiger Sicht. Zur Förderung des metalinguistischen Bewusstseins. In: Vetter, Eva (Hrsg.):

Professionalisierung für sprachliche Vielfalt. Perspektiven für eine neue LehrerInnenbildung. Baltmannsweiler: Schneider Hohengehren, 111-147

Bonnnet, Andreas \& Küppers, Almut (2011): Wozu taugen kooperatives Lernen und Dramapädagogik? In: Küppers, Almut; Schmidt, Torben; Walter, Maik (Hrsg.): Inszenierungen im Fremdsprachenunterricht: Grundlagen, Formen, Perspektiven. Hannover: Schroedel, 32-52

Brem, Christiane (2005): Theatererlebnisse. Mit Kindern Theaterstücke kreativ gestalten. Donauwörth: Auer

Chang, Li-Yu S. (2012): ,Dramatic ' Language Learning in the Classroom. In: Winston, Joe (Hrsg.): Second Language Learning through Drama. Practical Techniques and Applications. London/New York: Routledge, 6-14

Chang, Li-Yu S. \& Winston, Joe (2012): Using Stories and Drama to Teach EFL at Primary Level. In: Winston, Joe (Hrsg.): Second Language Learning through Drama. Practical Techniques and Applications. London/New York: Routledge, 15-30

Clark, Rachel, Morrison, Timothy G. \& Wilcox, Brad (2009): Readers' Theater: A process of developing fourth graders' reading fluency. In: Reading Psychology 30/4, 359-385

Corcoran, Carol A. \& Davis, A. Dia (2005): A study of the effects of Readers' Theatre on second and third grade special education students' fluency growth. In: Reading Improvement 42/2, 105-11

Cunningham, Anne E. \& Stanovich, Keith E. (1997): Early reading acquisition and its relation to reading experience and ability 10 years later. In: Developmental Psychology 33/6, 934-945

Deci, Edward L. \& Ryan, Richard M. (2000): The „What" and „Why“ of Goal Pursuits: Human Needs and the Self-Determination of Behavior. In: Psychological Inquiry 11/4, 227-268 
Eder, Ulrike (2015): „Alles gut“ für den DaZ- und Deutschunterricht. Exemplarische Literaturanalyse eines mehrsprachigen Bilderbuchs als Basis für mögliche Didaktisierungen. In: Eder, Ulrike (Hrsg.): Sprache erleben und lernen mit Kinder- und Jugendliteratur I. Theorien, Modelle und Perspektiven für den Deutsch als Zweitsprachenunterricht. Wien: Praesens

Europarat (2001): Gemeinsamer europäischer Referenzrahmen für Sprachen. Lehren, lernen und beurteilen.

http://www. goethe.de/z/50/commeuro/deindex.htm, letzter Zugriff

23.09.2015

European Commission (2012): Commission Staff Working Document. Language competences for employability, mobility and growth.

http://eur-lex.europa.eu/legal-

content/EN/TXT/PDF/?uri=CELEX:52012SC0372\&from=EN, letzter Zugriff 23.09.2015

Fratini, Nathalie (2008): Dramapädagogik im (Fremd)Sprachenunterricht. Eine wertvolle Unterstützung im Deutschunterricht in Luxemburg - Nicht nur für romanophone Sprachlerner. In: Scenario 2008/2, 41-50.

[http://research.ucc.ie/scenario/2008/02/fratini/04/de, letzter Zugriff: 23.09.2015]

Grabe, William (2014): Key Issues in L2 Reading Development. In: Deng, Xudong\& Seow, Richard (Hrsg.): Alternative Pedagogies in the English Language and Communication Classroom. 4th CELC Symposium Proceedings. Singapore: Centre for English Language Communication, National University of Singapore, 8-18

Haack, Adrian \& Surkamp, Carola (2011): „Theatermachen“ inszenieren Dramapädagogische Methoden in der Lehrerbildung. In: Küppers, Almut; Schmidt, Torben; Walter, Maik (Hrsg.): Inszenierungen im Fremdsprachenunterricht. Grundlagen, Formen, Perspektiven. Braunschweig: Schroedel, 53-66

Herdina, Philip \& Jessner, Ulrike (2002): A dynamic model of multilingualism: Perspectives of change in psycholinguistics. Clevedon: Multilingual Matters

Hilliger, Dorothea (2009): Theaterpädagogische Inszenierung. BeispieleReflexionen - Analysen. Berlin: Schibri

Kempston, Tanya (2012): Using Drama to Enrich School-Based Assessment in the Hong Kong Secondary School English Language Classroom. In: Winston, Joe (Hrsg.): Second Language Learning through Drama. Practical Techniques and Applications. London/New York: Routledge, 92-103

Kessler, Benedikt (2008): Interkulturelle Dramapädagogik. Dramatische Arbeit als Vehikel des interkulturellen Lernens im Fremdsprachenunterricht. Frankfurt a.M.: Lang

Küppers, Almut \& Schmidt, Torben; Walter, Maik (2011): Inszenierungen im Fremdsprachenunterricht. Grundlagen, Formen, Perspektiven. Hannover: Schroedel 
Maley, Alan (1995): Short and Sweet. Short Texts and How to Use Them. 2 Bd., London: Penguin

Maley, Alan \& Duff, Alan (1978/2005): Drama Techniques A resource book of communication activities for language teachers. $3^{\text {rd }} \mathrm{ed}$. Cambridge: Cambridge University Press

Martens, Gitta (2008): Wisst ihr, was gestern passiert ist? Kinder erzählen und spielen Playbacktheater in Grundschule und Freizeit. Weinheim: Deutscher Theaterverlag

Martinez, Miriam; Roser, Nancy L. \& Strecker, Susan (1998/1999): „I never thought I could be a star": Readers' Theatre in a tutorial for children with reading problems. In: The Reading Teacher 52/4, 326-334

Mraz, Maryann; Nichols, William; Caldwell, Safronia; Beisley, Rene; Sargent, Stephan \& Rupley, William (2013): Improving Oral Reading Fluency through Readers Theatre. In: Reading Horizons 52/2, 163-180

Nieveen, Nienke (2010): Formative Evaluation in Educational Design Resarch. In: Plomp, Tjeerd \& Nieveen, Nienke (Hrsg.): An Introduction into Educational Design Research. Eschede: SLO, 89-101

OECD (2011): PISA 2009 Ergebnisse: Potenziale nutzen und Chancengerechtigkeit sichern - Sozialer Hintergrund und Schülerleistungen. Band II. Paris: OECD. [http://dx.doi.org/10.1787/9789264095359-de, letzter Zugriff: 23.09.2015]

OECD (2014): PISA 2012 Ergebnisse: Was Schülerinnen und Schüler wissen und können. Schülerleistungen in Lesekompetenz, Mathematik und Naturwissenschaften. Band I, Überarbeitete Ausgabe, Februar 2014. Bielefeld: Bertelsmann. [http://dx.doi.org/10.1787/9789264208858-de, letzter Zugriff: 23.09.2015]

Plomp, Tjeerd (2010): Educational Design Research: An Introduction. In: Tjeerd, Plomp \& Nieveen, Nienke (Hrsg.): An Introduction into Educational Design Research. Eschede: SLO, 9-35

Rosebrock, Cornelia; Nix, Daniel; Rieckmann, Carola \& Gold, Andreas (2011): Leseflüssigkeit fördern. Lautleseverfahren für die Primar- und Sekundarstufe. Seelze: Klett/Kallmeyer

Schäfer, Martina (2006): Geschichten Erzählen (k)eine Kunst. In: Steinweg, Reiner (Hrsg.). Erzählen, was ich nicht weiß. Berlin: Schibri, 13-22

Schewe, Manfred (1993): Fremdsprache inszenieren. Zur Fundierung einer dramapädagogischen Lehr- und Lernpraxis. Oldenburg: Didaktisches Zentrum, Universität Oldenburg

Schewe, Manfred (2007): Drama und Theater in der Fremd- und Zweitsprachenlehre - Blick zurück nach vorn. In: Scenario 1/2007, 158-73. [http://publish-ucc.ie/journals/scenario/2007/01/schewe/08/de, letzter Zugriff: 01.09.2015]

Schewe, Manfred (2011): Die Welt auch im fremdsprachlichen Unterricht immer wieder zu verzaubern - Plädoyer für eine performative Lehr und 
Lernkultur. In: Küppers, Almut; Schmidt, Torben \& Walter, Maik (Hrsg.): Inszenierungen im Fremdsprachenunterricht. Grundlagen, Formen, Perspektiven. Hannover: Schroedel, 20-31

Schewe, Manfred; Shaw, Peter (Hrsg.) (1993): Towards Drama as a Method in the Foreign Language Classroom. Frankfurt am Main: Lang

Schmidt, Isolde (2008): Textorientierung - Schülerorientierung. In: Ahrens, Rüdiger; Eisenmann, Maria \& Merkl, Matthias (Hrsg.): Moderne Dramendidaktik für den Fremdsprachenunterricht. Heidelberg: Universitätsverlag Winter, 19-35

Swale, Jessica (2015): Drama Games for Classrooms and Workshops. London: Nick Hern Books

Tyler, Brenda-Jean \& Chard, David J. (2000): Using Readers Theatre to foster fluency in struggling readers: A twist on the repeated reading strategy. In: Reading and Writing Quarterly 16/2, 163-168

Van den Akker, Jan; Gravemeijer, Koeno; McKenney, Susan \& Nieveen, Nienke (Hrsg.) (2006): Educational Design Research. London: Routledge

Villiger, Caroline; Niggli, Aois; Wandeler, Christina; Watermann, Rainer \& Kutzelmann, Sabine (2010): Multiple Ziele bei der Leseförderung. Befunde aus einer vergleichenden Interventionsstudie auf Klassenstufe 4. In: Journal für Bildungsforschung Online (JERO) 2/2, 153-194

Vlcek, Radim (2006): Workshop Improvisationstheater. Übungs- und Spielesammlung für Theaterarbeit, Ausdrucksfindung und Gruppendynamik. Donauwörth: Auer

Volkmann, Laurenz (2008): Acting out: Möglichkeiten des darstellenden Spiels mit englischen Texten von der Unter- bis zu Oberstufe. In: Ahrens, Rüdiger; Eisenmann, Maria; Merkl, Matthias (Hrsg.): Moderne Dramendidaktik für den Fremdsprachenunterricht. Heidelberg: Universitätsverlag Winter, 425-449

Walk, Brigitte (2015): Handout und Literaturliste zum Seminar „Lesetheater und Theaterpädagogik" am 21.05.2015, Pädagogische Hochschule Vorarlberg. Unveröffentlichtes Manuskript

Wardetzky, Kristin (2007): Projekt Erzählen. Baltmannsweiler: Schneider Hohengehren

Winston, Joe (2012): Introduction: Second and Additional Language Learning through Drama. In: Winston, Joe (Hrsg.): Second Language Learning through Drama. Practical Techniques and Applications. London/New York: Routledge, 1-5

Worthy, Jo \& Prater, Kathryn (2002): „I thought about it all night“: Readers Theatre for reading fluency and motivation. In: The Reading Teache, 56/3, 294-198

Young, Chase \& Rasinski, Timothy (2009): Implementing Readers Theatre as an approach to classroom fluency instruction. In: The Reading Teacher 63/1, 4-13 